\title{
Editorial: From Ecology to Cancer Biology and Back Again
}

\author{
Frederick R. Adler ${ }^{1 *}$, Sarah R. Amend ${ }^{2}$, Etienne Baratchart ${ }^{3}$ and Christopher J. Whelan ${ }^{4,5}$ \\ ${ }^{1}$ Department of Mathematics, School of Biological Sciences, University of Utah, Salt Lake City, UT, United States, ${ }^{2}$ The \\ James Buchanan Brady Urological Institute, Johns Hopkins University School of Medicine, Baltimore, MD, United States, \\ ${ }^{3}$ Division of Translational Cancer Research, Lund Stem Cell Center, Lund University, Lund, Sweden, ${ }^{4}$ Department of Cancer \\ Physiology, Moffitt Cancer Center and Research Institute, Tampa, FL, United States, ${ }^{5}$ Department of Biological Sciences, \\ University of Illinois at Chicago, Chicago, IL, United States
}

Keywords: cancer, ecology, competition, community ecology, metastasis, evolution

\section{Editorial on the Research Topic}

\section{From Ecology to Cancer Biology and Back Again}

Application of evolutionary concepts to the study of cancer progression transformed cancer research in the 1970's (Cairns, 1975; Nowell, 1976) and inspired novel approaches to therapy. This work provided the framework for foundational discoveries in cancer genetics including those related to tumor cell heterogeneity, inherited risk of cancer, and synthetic lethality. Of the four classically defined forces of evolution (mutation, gene flow, genetic drift, and selection), however, only mutation is firmly classified in the field of genetics. The remaining three are ecological: gene flow depends on the movement of individuals, genetic drift on how population sizes vary in time and space, and selection on interactions with the biotic and abiotic environment.

Researchers are increasingly applying the ecological principles that underlie evolution to study cancer biology, appreciating that understanding the complex ecology of the tumor is essential to successfully treat this lethal disease. Conversely, the conceptual framework and quantitative tools from cancer biology have the potential to transform the understanding of the complexity of ecology itself, opening new ways to address the ecological challenges that define our times.

This Research Topic, therefore, was curated to support two goals.

Edited and reviewed by: Jordi Figuerola,

Doñana Biological Station, Spanish National Research Council (CS/C), Spain

*Correspondence: Frederick R. Adler adler@math.utah.edu

Specialty section: This article was submitted to Behavioral and Evolutionary Ecology, a section of the journal

Frontiers in Ecology and Evolution

Received: 21 December 2021

Accepted: 05 January 2022

Published: 10 February 2022

Citation:

Adler FR, Amend SR, Baratchart E and Whelan CJ (2022) Editorial: From Ecology to Cancer Biology and Back Again. Front. Ecol. Evol. 10:840375.

doi: 10.3389/fevo.2022.840375
1) Integrate ecosystem, behavioral, and physiological ecology into the study of cancer. The evolution that leads to resistance and metastasis is driven not only by the heterogeneity and phenotypic interactions that are the purview of ecology focused on the species level, but also by the flows of energy, materials and nutrients, and the changing phenotypes of individuals.

2) Use the insights of cancer ecology to rethink ecology itself, in particular by using modern molecular and genetic tools to address core questions and to apply them to forecasting and restoration.

\section{FROM THE CORE ISSUES IN ECOLOGY TO CANCER AND BACK AGAIN}

Many of the papers in this Research Topic focus on the core issues that have defined the science of ecology since its founding: persistence of species, the maintenance of diversity and coexistence, and the distribution and abundance of species and their interactions.

Interactions among cancer cells and those between cancer cells and host cells display many ecological processes, including niche construction, resource exploitation (Huntly et al.; Kareva and Brown; Somarelli; Wu), predator-prey interactions (Peplinski et al.; Somarelli), source-sink population dynamics (Cunningham et al.), game-theoretic interactions among different cancer cell types (Kareva and Brown; Noble et al.; Pressley et al.), and hijacking of signaling mechanisms, including those governing metabolic pathways and immunological defenses (Bukkuri and Adler). 
These interactions often involve the tradeoffs that support coexistence of different tumor cell types (Huntly et al.; Kareva and Brown). In relation to ecological management, control of tumors parallels the control of invasive species (Neinavaie et al.) and combinations of approaches may be designed to drive the extinction of cancer (Gregg).

\section{FROM PHENOTYPES TO ECOLOGY AND BACK AGAIN}

Advancing technological methodology has enabled the identification of individual cell phenotypes, including physiological states that determine cell behaviors and interactions among cancer cells and between cancer cells and the tumor microenvironment. This concept underpins tumor microenvironment research, and highlights the power of ecological concepts to understand tumor biology, progression, and lethality (Bissell and Hines, 2011; Myers et al., 2020). In ecology, dormancy plays a key role in the life histories of organisms that deal with unpredictable environments, and this phenotypic state is often observed clinically prior to cancer recurrence (Kostecka et al.; Miller et al.). While these similarities are informative, there are key differences between cancer cell and animal dormancy, with cancer cells often altering rather than simply slowing metabolism. These reversible metabolic changes may help cancers survive fluctuating resources and promote expensive but flexible glycolysis over or simultaneous with oxidative phosphorylation (Huntly et al.). These metabolic differences reveal themselves in scaling relationships between size and metabolism, with tumors showing strong and consistent scaling relationships that differ from healthy tissue, such as a high volumetric scaling factor that reflects the high resource needs of tumors (Brummer and Savage). These changes in metabolism can interact with ecological factors to alter the foraging behavior and growth of mice with cancer (Makin et al.). Differences in cell phenotypes can generate novel interactions. For example, one cancer cell population can exploit another one, increasing its fitness to the detriment of the other (Noble et al.).

\section{FROM ECOLOGY TO EVOLUTION AND BACK AGAIN}

Despite the underlying stochasticity of mutation, some aspects of cancer are quite predictable, with cancers showing convergent evolution on the hallmarks of cancer by quite different mechanisms due to selection driven by the ecology of the cancer (Somarelli). On the other hand, predicting cancer evolutionary dynamics at the patient level remains quite challenging. Although numerous genome sequencing studies have offered some understanding of the cancer clonal makeup, the lack of phenotypic characterization of the tumor composition can make interpretation difficult (Plutynski). The ecological factors that drive cancer evolution can provide the crucial bridge. As an example, the rapid evolution induced in human-dominated ecosystems by harvesting and habitat change parallels the selection placed on cancer by treatment, generating the full range of qualitative and quantitative resistance (Pressley et al.).
Resource availability also creates strong selection, with evidence supporting roles of high availability of glucose, iron, or phosphate promoting aggressive cancer growth $(\mathrm{Wu})$. Cancer cells are well-known to tolerate low oxygen levels, and this potential may reflect the role of hypoxia in shaping cell differentiation during development, and argue that cancer cells might face challenges in overcoming the evolutionary legacy to retain stem like characteristics during normoxia (Carroll et al.).

Studies of evolution often focus on mutations. However, polyploidization and chromosomal instability lead to the accession of the polyaneuploid cancer cell (PACC) state that enables dormancy, treatment escape, and relapse initiation (Kostecka et al.). Changes in cell motility and collective behavior underlie metastasis, the deadliest phenotype of solid tumors. Parallels between metastasis and invasion in ecology may elucidate how metastatic cells colonize and adapt to foreign soil (Neinavaie et al.), pointing a way to understand and predict their spread.

\section{FROM EVOLUTION TO THE PREVENTION AND TREATMENT OF CANCER AND BACK AGAIN}

Application of ecological and evolutionary principles has the potential to directly transform cancer patient care in diagnostics (Maley et al., 2017) and novel treatment strategies (Pienta et al., 2008; Amend and Pienta, 2015; Whelan and Gatenby, 2020). Indeed, many successful anti-cancer treatment strategies (e.g., bone marrow transplant in leukemias, immunotherapy checkpoint inhibitors in many solid tumors) are truly tumor ecology-informed therapeutic strategies. Specific application of an eco-evolutionary framework will identify other avenues for treatment innovation. Elucidating how metabolic needs shape cancer evolution implicates patient diet and lifestyle in cancer risk $(\mathrm{Wu})$ and suggests dietary therapies that could complement chemotherapy and other conventional treatments (Gregg). Ecoevolutionary principles open up new therapeutic opportunities based on competition (Pressley et al.; Somarelli), dormancy (Kostecka et al.), predation (Peplinski et al.), and positive cell interactions (Noble et al.).

\section{FROM QUESTIONS TO CONCLUSIONS AND BACK AGAIN}

Several themes and challenges emerge from this collection. First, the study of cancer cells in a tumor gives new insight into the regulation of healthy systems (Bukkuri and Adler). The cancer cells in a tumor demand and use resources like individuals in ecology (Cunningham et al.; $\mathrm{Wu}$ ), and those resources include signaling molecules like hormones that mirror signals between individuals as in populations of plants (Kareva and Brown). Placing cancers in the larger biological context of evolution and development makes sense of properties like tolerance of hypoxia (Carroll et al.). Like species, cancer cells can, at least hypothetically, go extinct well-before their hosts (Gregg).

Second, heterogeneity matters. Individuals, cells, locations, and times differ (Huntly et al.), and these differences feedback 
to affect each other (Gregg). The mechanisms underlying heterogeneity, can be genetic and heritable (Neinavaie et al.) or plastic and epigenetic (Gregg). Third, evolution and ecology are intimately linked. As in nature, evolution in cancer is driven by ecological interactions among cancer cells and between cancer cells and cells of the host, including fibroblasts, immune cells, the associated vasculature, and many other components of the tumor microenvironment.

The levels of selection created by population sub-structuring are critical from the perspective of the patient and the treating oncologist. For example, the success of temporal escape through dormancy depends on the behaviors of the individual cancer cells (Kostecka et al.; Miller et al.). Rates of evolution determine the ability to respond to novel selection regimes, and, in addition to mutation rates, these rates emerge from population dynamics (Pressley et al.). The efficiency of selection depends on population size and population structure. As in ecology, it is important to consider that observed traits may not be adaptive, but rather may result from drift and past competition.

The type of data and feasible experiments in ecology and cancer biology differ. With cancer patients, interventions and measurements must be carefully designed to minimize potential harm, while in ecological systems we are often constrained by feasibility, environmental protection, and funding. Laboratory and greenhouse systems sacrifice realism for control and may simulate pulsed resource environments that select for "creamskimmer" phenotypes (Huntly et al.). Prior hypotheses can lead to publication bias of finding mainly what we are looking for $(\mathrm{Wu})$. Ultimately, cancer biology rigor and ecological rigor are different but can and should be combined in cancer ecology (Plutynski). Both fields bring qualities that the other lacks: cancer biology is focused on molecular and cellular mechanisms underlying observed phenotypes, while ecologists unravel causal networks in complex systems that have been shaped by their evolutionary history.

\section{REFERENCES}

Amend, S. R., and Pienta K. J. (2015). Ecology meets cancer biology: the cancer swamp promotes the lethal cancer phenotype. Oncotarget 6, 9669-9678. doi: 10.18632/oncotarget.3430

Bissell, M. J., and Hines W. C. (2011). Why don't we get more cancer? A proposed role of the microenvironment in restraining cancer progression. Nat. Med. 17, 320-329. doi: 10.1038/nm.2328

Cairns, J. (1975). Mutation selection and the natural history of cancer. Nature 255, 197-200. doi: 10.1038/255197a0

Maley, C. C., Aktipis, A., Graham, T. A., Sottoriva, A., Boddy, A. M., Janiszewska, M., et al. (2017). Classifying the evolutionary and ecological features of neoplasms. Nat. Rev. Cancer 17, 605-619. doi: 10.1038/nrc.2 017.69

Myers, K. V., Pienta, K. J., and Amend, R. S. (2020). Cancer cells and M2 macrophages: cooperative invasive ecosystem engineers. Cancer Control. 27:1073274820911058. doi: 10.1177/10732748209 11058

Nowell, P. C. (1976). The clonal evolution of tumor cell populations. Science 194, 23-28. doi: 10.1126/science.959840

Pienta, K. J., McGregor, N., Axelrod, R., and Axelrod, E. D. (2008). Ecological therapy for cancer: defining tumors using an ecosystem paradigm suggests new opportunities for novel cancer treatments. Transl. Oncol. 1, 158-164. doi: $10.1593 /$ tlo. 08178
Finally, as in all science, we must think about the goals and appropriate scale of inquiry of any biological investigation, whether the ultimate aim is fundamental understanding, forecasting, or treatment of disease. If we observe ecological phenomena like facilitation and competition (Noble et al.) or predation (Peplinski et al.) in cancer, do we need to understand the molecular details to effectively harness these phenomena for developing new treatment strategies? As genetic and molecular methods continue to unify the sciences, we think that the crosstalk between the methods, questions, and approaches between cancer ecology and traditional ecology will continue to increase to the benefit of both fields.

\section{AUTHOR CONTRIBUTIONS}

FA outlined and drafted the initial version and edited later drafts. SA, EB, and CW contributed to the writing and editing. All authors contributed to the article and approved the submitted version.

\section{FUNDING}

SA is supported by US Department of Defense CDMRP/PCRP (W81XWH-20-10353), the Patrick C. Walsh Prostate Cancer Research Fund, and the Prostate Cancer Foundation. CW was supported by NIH/NCI PSOC U54CA193489, NIH/NCI P30CA076292, and NIH/NCI U54 Supplement. FA was supported by U54 CA209978 NCA NIH and NIH U01 CA264620.

\section{ACKNOWLEDGMENTS}

We thank the authors for their inspiring contributions to this Research Topic.

Whelan, C. J., and Gatenby, R. A. (2020). Special collection on ecological and evolutionary approaches to cancer control: cancer finds a conceptual home. Cancer Control 27:1073274820942356. doi: 10.1177/1073274820942356

Conflict of Interest: SA holds equity interest in Keystone Biopharma, Inc.

The remaining authors declare that the research was conducted in the absence of any commercial or financial relationships that could be construed as a potential conflict of interest.

Publisher's Note: All claims expressed in this article are solely those of the authors and do not necessarily represent those of their affiliated organizations, or those of the publisher, the editors and the reviewers. Any product that may be evaluated in this article, or claim that may be made by its manufacturer, is not guaranteed or endorsed by the publisher.

Copyright (c) 2022 Adler, Amend, Baratchart and Whelan. This is an open-access article distributed under the terms of the Creative Commons Attribution License (CC $B Y)$. The use, distribution or reproduction in other forums is permitted, provided the original author(s) and the copyright owner(s) are credited and that the original publication in this journal is cited, in accordance with accepted academic practice. No use, distribution or reproduction is permitted which does not comply with these terms. 